\title{
Gleb Wataghin *
}

\author{
R. A. SALMERON
}

É

COM GRANDE honra e imenso prazer que tenho a oportunidade de falar a respeito de Gleb Wataghin, o homem que ensinou aos brasileiros os fundamentos da física moderna, como aprender esses fundamentos e como fazer pesquisa em física. A história de Gleb Wataghin no Brasil é uma história de grande beleza e fora do comum. Não há muitos exemplos semelhantes de cientistas que, por suas ações pessoais, tiveram tão grande influência sobre tantas pessoas de gerações diferentes num país, que nem era o seu próprio país. Os alunos de Gleb Wataghin, e os alunos de seus alunos, espalharam-se por diferentes lugares, contribuindo para fazer da física brasileira o que ela é hoje.

A descrição do trabalho de Gleb Wataghin no Brasil necessitaria muito mais do que uma simples palestra. Poderemos, entretanto, apresentar um pequeno resumo no qual tentaremos pôr em evidência alguns dos aspectos da sua personalidade como homem, como físico e como mestre.

\section{A fundação da Universidade de São Paulo}

Gleb Wataghin nasceu na Rússia, mas viveu na Itália, para onde sua família emigrou. Estudou naquele país e, em 1934, veio de Turim para São Paulo. Para compreender a influência que exerceu, e poder avaliar a sua extensão, é necessário conhecer algo a respeito das escolas superiores no Brasil naquele período. Havia no país uma só universidade, fundada em 1920 no Rio de Janeiro. Esta universidade era formada pelas escolas superiores já existentes antes da sua fundação: Engenharia, Medicina, Direito e Odontologia, colocadas então sob uma única administração, mas sem qualquer conexão - intelectual ou profissional - entre elas. Em vários lugares no Brasil havia escolas superiores isoladas equivalentes a essas, mas que também não mantinham ligação alguma entre elas.

Não havia no país escolas especializadas no estudo das ciências da natureza, ciências humanas ou literatura. Em geral, as pessoas interessavam-se por matemá-

* Este artigo foi escrito pelo autor originalmente em inglês, como transcrição de uma conferência pronunciada na comemoração do centenário do nascimento de Gleb Wataghin, no evento que foi ao mesmo tempo o XI International Symposium on Very High Energy Cosmic Rays Interactions - chamado Edição do Centenário de Gleb Wataghin na série do Simpósio - e VI Gleb Wataghin School on High Energy Phenomenology, realizado na Universidade Estadual de Campinas, Campinas, de 17-21 jul. 2000. 
tica, física ou química quando estudavam engenharia; os que se interessavam por biologia, estudavam medicina; os especialistas em literatura, vinham principalmente das escolas de direito, e assim por diante. Os cientistas de quase todos os campos da ciência, os filósofos, os escritores, adquiriam a maior parte da sua educação profissional como autodidatas. Pouquíssimos tinham a oportunidade de aprimorar a sua formação na Europa.

Essa era a situação no Brasil quando a Universidade de São Paulo foi fundada, em 1934, reunindo as escolas superiores que havia na cidade sob uma mesma administração. Mas, mais importante do que a junção das escolas do ponto de vista administrativo foi a criação da Faculdade de Filosofia, Ciências e Letras da USP, a primeira fundada no país. Esta Faculdade teve enorme impacto sobre o desenvolvimento das ciências em São Paulo, com subseqüente influência sobre outras universidades fundadas posteriormente. Devido a esta influência, a fundação da Universidade de São Paulo foi o acontecimento mais importante na história das universidades brasileiras.

Os fundadores da USP decidiram que eminentes professores europeus deveriam ser convidados para começar o ensino e a pesquisa nas diferentes ciências. A organização dos convites foi confiada ao insigne matemático brasileiro Teodoro Ramos, que havia trabalhado durante vários anos em Paris, na Sorbonne, e mantinha bom relacionamento com a comunidade científica européia. Teodoro Ramos escreveu um dos primeiros livros sobre análise vetorial em língua francesa, quando trabalhava em Paris. A sua influência na organização da nova universidade foi enorme, por ele ter convidado pessoas do mais alto nível para iniciar ciências humanas, ciências da natureza e matemática na recém-fundada universidade. Foi a Roma formalizar convite a Enrico Fermi para dirigir a física. Fermi, naquela época, estava envolvido com os famosos experimentos que se tornaram históricos, e não pôde aceitar o convite. Recomendou Gleb Wataghin, que então trabalhava na Universidade de Turim, um dos físicos teóricos italianos em quem Fermi, segundo suas próprias palavras, tinha confiança. Wataghin aceitou o convite para vir ao Brasil.

\section{Wataghin o homem}

A decisão de Wataghin foi uma grande oportunidade para o Brasil. Olhando retrospectivamente para a história, vemos que dificilmente se poderia encontrar pessoa mais adequada para começar a física em São Paulo, não somente por sua estatura científica, mas também por suas qualidades humanas. Para iniciar a pesquisa em física num país sem tradição nesse campo, ele teve de enfrentar dificuldades de diferentes tipos: humanas, culturais, administrativas, financeiras. Resolveu-as sempre com energia, sabedoria e tato. 
Wataghin era homem jovial, sorridente, com amplo interesse pelas coisas da vida e pelo relacionamento humano. Apaixonado pela ciência, gostava de ensinar, e estava sempre disponível para longas palestras com os estudantes.

Muitas vezes, no meio de uma conversa a respeito da física, ele dissertava sobre a contribuição para a ciência de "pessoas de pensamentos profundos", exprimindo a sua admiração pessoal por muitos dos físicos seus contemporâneos. Essas conversas estimulavam nos jovens a curiosidade pela ciência e o respeito pelos grandes cientistas.

Uma das principais qualidades de Wataghin como mestre era a sua fé nos jovens. Sempre entusiasta sobre a evolução dos jovens cientistas, costumava dizer que, no seu progresso contínuo, de repente eles desabrochavam "como uma flor".

Gleb Wataghin, e outros professores europeus que vieram para a Faculdade de Filosofia, Ciências e Letras da USP, introduziram a possibilidade de se mandar jovens para o exterior, para trabalharem com eminentes pesquisadores em grandes centros universitários.

O respeito que tinha pelos seus estudantes e o nível que desejava imprimir ao Departamento de Física da nova Universidade podem ser avaliados quando se conhece quem eram os físicos escolhidos como orientadores de seus estudantes quando iam para o exterior, depois de terem trabalhado com ele por dois ou três anos. Wataghin enviou Marcello Damy de Souza Santos, físico experimental, para trabalhar com William Bragg, na Universidade de Cambridge, que recebera o Prêmio Nobel de Física (PNF); Souza Santos tornou-se um dos líderes da física brasileira, ao construir um acelerador de eletrons do tipo betatron. Foi com ele que se iniciou a física com aceleradores e a física nuclear no Brasil. Instalou e pôs em operação o primeiro reator nuclear no país e fundou o Instituto de Pesquisas em Energia Nuclear.

Paulus Aulus Pompéia, também físico experimental, sob a orientação de Arthur Compton (PNF) na Universidade de Chicago, tornou-se outro líder no campo da física no Brasil, tendo sido um dos principais organizadores do Instituto Tecnológico de Aeronáutica, em São José dos Campos, uma das mais importantes escolas de engenharia do país.

Mário Schenberg, físico teórico, foi trabalhar com o grupo de Enrico Fermi (PNF) na Universidade de Roma; Sônia Ashauer, física teórica, teve como orientador Paul Dirac (PNF), na Universidade de Cambridge (Ashauer infelizmente faleceu precocemente); Walter Schutzer (que também faleceu jovem), trabalhou com Eugene Wigner (PNF) na Universidade de Princeton; Jayme Tiomno recebeu sua orientação de John Wheeler e Eugene Wigner, em Princeton; Paulo Leal Ferreira trabalhou com o grupo de Roma e, com seu irmão Jorge, fundou o Instituto de 
Física Teórica em São Paulo, um dos mais importantes do país. Schenberg, Tiomno e Leal Ferreira estão entre os líderes mais proeminentes da física teórica no Brasil.

Oscar Sala trabalhou com R.G. Herb, na Universidade de Wisconsin, e é um dos mais destacados líderes da física nuclear no país. Fundou o Departamento de Física Nuclear da USP, com laboratório moderno bem equipado com aceleradores van de Graaff.

Outro estudante de Wataghin que se tornou muito conhecido é Cesar Lattes, que trabalhou com Cecil Powell (PNF), na Universidade de Bristol, principalmente devido a suas ligações com Giuseppe Occhialini. Lattes fundou, no Rio de Janeiro, o Centro Brasileiro de Pesquisas Físicas e, posteriormente, iniciou as pesquisas em raios cósmicos na Unicamp.

\section{Wataghin o físico}

Wataghin trabalhava em física teórica e em física experimental, qualidade excepcional que foi extremamente importante para o começo da física em São Paulo. Assim, desde seu início a física contou com pessoas formadas nos dois campos - o teórico e o experimental. Pertenceu àquela última geração de poucos físicos que dominavam inteiramente seu campo de atividade. Podia ministrar de improviso, sem preparo prévio, uma aula sobre praticamente qualquer ramo da física do seu tempo.

A sua vasta cultura era complementada por uma intuição fora do comum para fenômenos físicos, o que lhe permitia predizer, às vezes com grande antecedência, o futuro de uma linha de pesquisa ou de um experimento recém-começado. Gostaria de citar dois exemplos de conversas que tivemos. Uma delas foi no CERN, em Genebra, quando lhe mostrei pela primeira vez os preparativos de experimentos que iríamos realizar sobre interações de neutrinos. Bastaram poucos minutos para que ele concluísse quais as variáveis importantes que deveríamos medir para fornecer elementos à teoria, e que aqueles experimentos poderiam mudar alguns dos nossos conceitos em física. De fato, eles forneceram as primeiras evidências que nos levaram a pensar seriamente sobre a realidade de QCD. Em outra conversa, quando o informei que pares de muons tinham sido produzidos em colisões, Wataghin concentrou-se por alguns minutos, dizendo em seguida que pares de muons poderiam provir de raios gama, como os pares de eletrons, e formulou duas perguntas: de onde viriam os raios gama em colisões hadrônicas, e se deveríamos investigar a produção de raios gama nessas colisões. Ele tinha razão, sabemos hoje que o mecanismo é a produção de um raio gama virtual pela aniquilação de um quark com um antiquark, com subseqüente desintegração do raio gama em pares de muons, de modo semelhante à aniquilação eletron-positron em QED. Processo importante para nossa compreensão dos quarks. 


\section{Física teórica}

Gleb Wataghin trabalhou em diversos ramos da física teórica:

- teoria dos campos, com ênfase em teorias de campos não-locais, assunto sobre o qual produziu um artigo pioneiro em 1934, ao mesmo tempo que Yukawa; ele esteve sempre interessado na estrutura espaço-tempo da teoria e, durante toda a sua vida, convencido de que deveria existir um comprimento fundamental nas interações;

- estatística de partículas a altas temperaturas;

- astrofísica (composição das estrelas);

- produção múltipla de mesons;

- teoria não-local de quarks com componentes.

\section{Modelo estatístico de produção de mesons}

Descreveremos sumariamente, como um exemplo de seus trabalhos, o modelo estatístico de Wataghin de produção de mesons em colisões de altas energias, que ele desenvolveu em 1941-1942, antes da descoberta do pion. Tanto quanto sabemos, este foi o primeiro modelo estatístico de produção de partículas.

A percepção física e a intuição de Wataghin podem ser avaliadas por suas próprias palavras ao descrever o modelo: "a finalidade das observações que seguem é mostrar alguns aspectos muito simples das distribuições de energia e de quantidade de movimento num grupo de partículas criadas em colisões de altas energias (...) Examinemos a colisão de alta energia de dois nucleons no sistema do centro de massa (...) começando com a hipótese de que quase toda a perda de energia é radiada sob a forma de um campo mesônico e que o número correspondente de mesons é grande, podemos tentar aplicar considerações clássicas à colisão, lembrando que neste caso os operadores que representam o campo mesônico quase comutam. Podemos então dizer que durante o tempo de colisão $\Delta t$, que é obviamente da ordem de $r_{0} / c$, onde $r_{0}$ é o alcance das forças nucleares, produz-se um pacote de onda que tem dimensão linear $r_{0}$. A análise de Fourier deste pacote contém termos que correspondem ao período $\Delta t$. Segue-se do princípio mencionado acima (1) que o comprimento de onda médio das ondas de mesons produzidas é também da ordem de $r_{0}$. (...) Nós encontramos que, na distribuição mais provável, os mesons criados têm, no sistema do centro de massa da colisão, energia $3 m c^{2}$ (onde $m$ é a massa em repouso do meson). (...) As conclusões gerais são: para altas energias $(\gamma>>1)$ o número $n$ de mesons e a energia média total dos mesons são proporcionais à raiz quadrada da energia primária (no sistema do centro de massa)" (2). 
Resumindo, Wataghin chegou a duas conclusões:

- A energia média dos mesons no sistema do centro de massa da colisão deve ser $<E_{m e s o n c m}>\wedge m c^{2}$, onde $m$ é a massa do meson. Sabemos hoje que isto é correto.

- A multiplicidade média dos mesons produzidos deve variar com a energia segundo $\left(E_{c m}\right)^{1 / 2}$ onde $E_{c m}$ é a energia da colisão no sistema do centro de massa.

Uma terceira conclusão, que Wataghin não mencionou explicitamente, é que a quantidade de movimento transversal do meson, $P_{T}$, deve ser limitada. Sabemos que isto é verdadeiro, o $P_{T}$ de pions produzidos em colisões de altas energias élimitado a $350-400 \mathrm{MeV} / c$, que é aproximadamente três vezes a massa do pion.

Gostaríamos de realçar que Wataghin foi um dos primeiros físicos a compreender que as colisões devem ser analisadas no sistema do centro de massa.

Notemos que Wataghin chegou a essas conclusões há 60 anos, numa época em que o pion ainda não tinha sido descoberto e a física de altas energias, isto é, a física de partículas elementares, ainda não existia. Sabemos hoje que a multiplicidade varia segundo $\log E_{c m}$. Esta variação é muito próxima da predição de Wataghin. Para comparação, a tabela apresentada a seguir dá a razão entre a multiplicidade média $\langle n>$ para certo valor da quantidade de movimento da partícula incidente no laboratório, $P_{l a b}$, e a multiplicidade média para a quantidade de movimento incidente igual a $10 \mathrm{GeV} / \mathrm{c}$, com as duas leis de variação: $\left(E_{c m}\right)^{1 / 2}$, como predito por Wataghin, e $\log E_{c m}$, como conhecemos atualmente.

$$
<\mathrm{n}>/<\mathrm{n}>\text { a } 10 \mathrm{GeV} / \mathrm{c}
$$

\begin{tabular}{ccc}
\hline$P_{l a b} G e V / c$ & $\left(E_{c m}\right)^{1 / 2}$ & $\log E_{c m}$ \\
\hline 10 & 1 & 1 \\
200 & 2.1 & 2.0 \\
500 & 2.6 & 2.3 \\
1000 & 3.1 & 2.5 \\
\hline
\end{tabular}

Pelos dados da tabela, percebe-se que a predição de Wataghin é muito próxima dos valores conhecidos atualmente; até à altíssima energia de $1000 \mathrm{GeV}$ a concordância é razoável. 


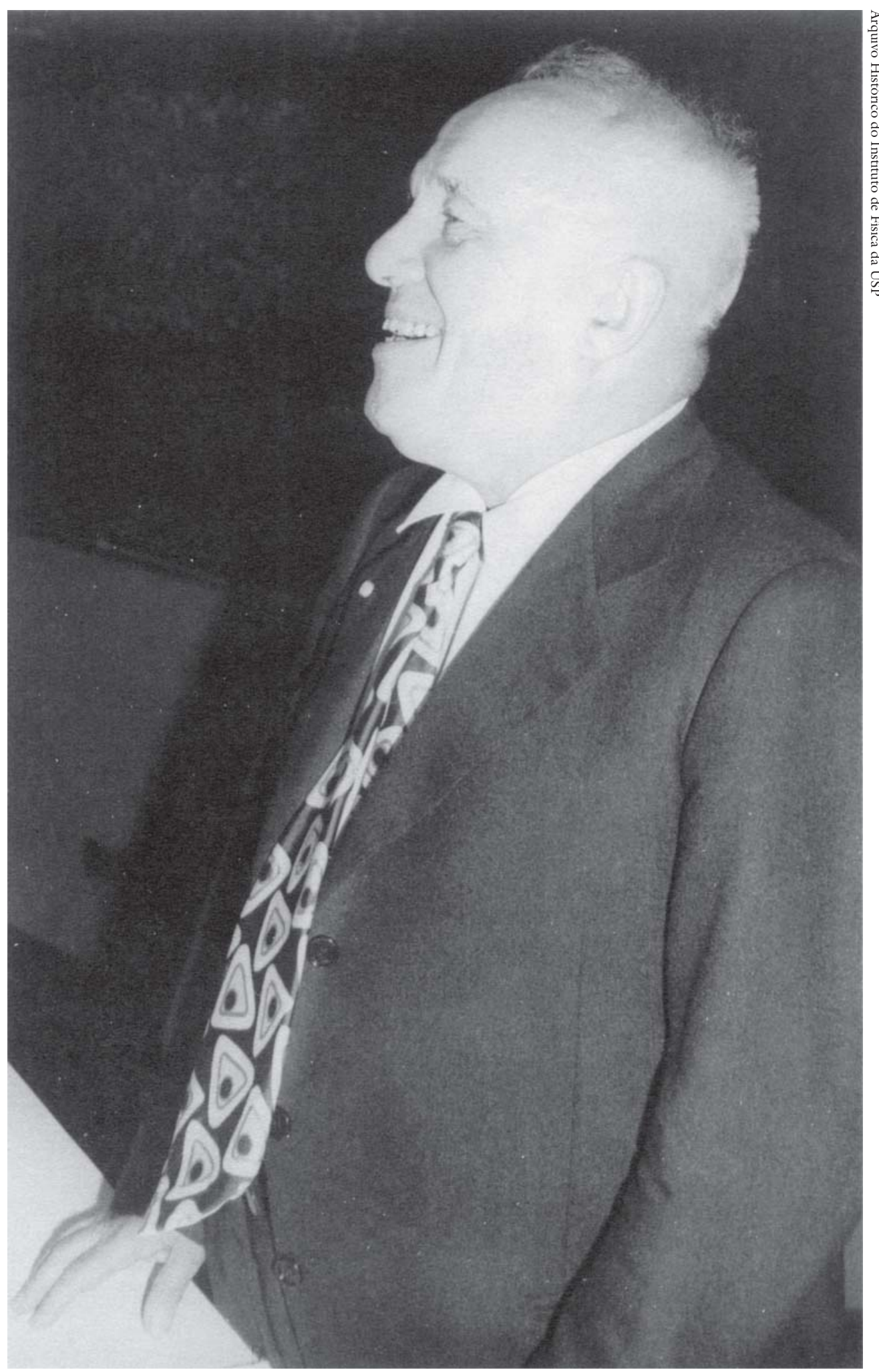

Gleb Wataghin em visita à Universidade Estadual de Campinas (Unicamp) em 1982 


\section{Pesquisa em raios cósmicos}

Wataghin teve perspicácia para logo compreender que o estudo de raios cósmicos era um campo de investigação importante, e que poderia ser estudado em boas condições no Brasil, na época em que aqui chegou. Essa sua visão marcou o desenvolvimento de certos setores da física brasileira, em alguns casos até hoje. Apesar de ser físico teórico, não tendo recebido qualquer formação especial em métodos experimentais, ele criou em São Paulo um grupo experimental em raios cósmicos que, em poucos anos, se tornou conhecido internacionalmente. Como exemplos do sucesso alcançado pelo grupo de São Paulo, citaremos dois de seus resultados: a descoberta dos chuveiros hadrônicos e a medida da seção de choque total de interação proton-proton a altas energias.

\section{A descoberta dos chuveivos hadrônicos}

Em 1940, Paulus A. Pompéia, M. Damy de Souza Santos e G. Wataghin fizeram em São Paulo um experimento que detectou eventos inesperados: chuveiros de partículas que poderiam atravessar dezenas de centímetros de chumbo (3). Naquela época eram conhecidos os chuveiros eletromagnéticos, mas estes podiam ser parados em poucos centímetros de chumbo. Os chuveiros detectados, que atravessavam dezenas de centímetros, não poderiam, portanto, ser eletromagnéticos. Eram de um novo tipo, que os autores chamaram "chuveiros penetrantes". Eles são o que denominamos hoje de "chuveiros hadrônicos", grupos de hadrons produzidos juntos em interações fortes. Os chuveiros hadrônicos são um dos elementos mais importantes em colisões de altas energias. Todos os grandes detectores de partículas utilizados em experimentos a altas energias precisam ser detectores de chuveiros hadrônicos.

Esta descoberta, além de ser relevante em si, teve resultados importantes, descritos, por exemplo, no livro de Heisenberg sobre Raios Cósmicos, que mostra uma conseqüência teórica e uma experimental.

A conseqüência teórica foram as tentativas para responder à pergunta se poderiam muitos mesons serem produzidos simultaneamente numa colisão, ou se eles seriam produzidos individualmente, isto é, somente um meson por colisão em muitas colisões sucessivas. $\mathrm{O}$ primeiro mecanismo, muitos mesons produzidos juntos, chamado de "produção múltipla", teve como seus mais ilustres defensores Heisenberg e Wataghin. O segundo mecanismo, pelo qual um único meson seria produzido numa colisão e o chuveiro o resultado de muitas colisões sucessivas, foi denominado "produção plural", e o seu mais proeminente defensor foi Heitler. Sabemos hoje que a hipótese de produção múltipla é a correta, mas tivemos de esperar por 15 anos, depois que o experimento de São Paulo foi realizado, para se ter a prova experimental, obtida com a produção de mesons em interações 
proton-proton numa câmara de Wilson no acelerador de Brookhaven.

A conseqüência experimental que queremos abordar foi o estímulo para a realização de outro experimento importante. Patrick Blackett (PNF), um dos mais eminentes físicos especialistas em raios cósmicos, diretor do Departamento de Física da Universidade de Manchester, na Inglaterra, influenciado pelo experimento de São Paulo, depois da Segunda Guerra Mundial sugeriu a G. Rochester e C. Butler que fizessem um experimento sobre chuveiros penetrantes, utilizando como detector uma câmara de Wilson imersa num campo magnético. O experimento foi realizado, e descobriu-se um novo tipo de partículas, até então desconhecidas, que foram denominadas "partículas V", porque elas se desintegram em duas outras partículas cujos traços formam um V na câmara de Wilson. Posteriormente o nome foi trocado para "partículas estranhas". Como é bem conhecido, esta descoberta produziu uma revolução na física.

\section{Seção de choque total proton-proton a altas energias}

Em 1945, Oscar Sala e Gleb Wataghin fizeram um experimento para estudar interações de raios cósmicos em parafina, que é rica em hidrogênio. Eles concluíram que a seção de choque total de interação proton-proton a altas energias deveria ser 40 milibarns (4), resultado extraordinariamente correto, obtido há 55 anos com um dispositivo experimental extremamente simples. Com resultados precisos de experimentos efetuados com aceleradores, sabemos que a altas energias esta seção de choque varia somente cerca de $10 \%$ em torno de 40 milibarns.

Gleb Wataghin retornou à Itália depois de ter trabalhado em São Paulo durante 16 anos. Realizou novamente um belíssimo trabalho na Universidade de Turim, com jovens físicos teóricos e experimentais, estes últimos tendo participado ativamente em muitos experimentos na "época de ouro" da física de partículas feita com raios cósmicos, os quais posteriormente passaram a trabalhar com aceleradores.

Este curto resumo de algumas das numerosas atividades de Gleb Wataghin e de alguns aspectos da sua personalidade é um tributo modesto a um grande homem que, partindo de nada num ambiente sem a mínima tradição em pesquisas físicas, teve forte influência sobre toda uma geração de cientistas e deu forma à física brasileira.

Notas

$1 \mathrm{O}$ princípio aqui mencionado tinha sido postulado por Wataghin, que sugeriu a invariança das leis da física em relação a uma transformação de "observáveis" e "representativos" de um estado na mecânica clássica e quântica. Cf. G. Wataghin, Nature 
393, n. 142, 1938; Comptes Rendues de l'Académie des Sciences, 358, n. 207, 1938 e 421, n. 207, 1938; Anais da Academia Brasileira de Ciências, jul. 1942.

2 G. Wataghin, On the production of groups of mesotrons by high energy collisions, em Symposium on Cosmic Rays, Rio de Janeiro, 1941, Anais da Academia Brasileira de Ciências, 1942, p. 129. Outras referências sobre o modelo estatístico são: G. Wataghin, Statistical mechanics of processes observed in cosmic ray phenomena, Anais da Academia Brasileira de Ciências, 355, n. 15, 1943; G. Wataghin, Statistical mechanics at extremely high temperatures, Phys. Rev. 149, n. 66, 1944; G. Wataghin, On the multiple production of mesons, Phys. Rev. 975, n. 74, 1948.

3 P.A. Pompéia, M.D. Souza Santos e G. Wataghin, Phys. Rev. 61, n. 57, 1940; Phys. Rev. 339, 57, 1940; Phys. Rev. 902, n. 59, 1941.

4 O. Sala e G. Wataghin, Phys. Rev. 5, n. 67, 1945.

R. A. Salmeron atua no Laboratoire de Physique Nucléaire des Hautes Energies, Ecole Polytechnique - 91128 Palaiseau Cedex - France. 